\title{
Design of a low cost, high performance PV inverter
}

\author{
T. T. $\mathbf{M a}^{\mathrm{a})}$ \\ Department of Electrical Engineering, CEECS, National United University, \\ 1 Lien-Da, Kung-Ching Li, Miaoli36003, Taiwan R.O.C. \\ a)tonyma@nuu.edu.tw
}

\begin{abstract}
The conventional grid-connected photovoltaic (PV) inverter that steps up low DC voltage to high DC voltage and cascades with the high frequency inverter is complicated in control and of low efficiency due to two stages. This paper presents a novel PV inverter system formed by a hybrid DC-DC converter and a full-bridge DCAC converter. The hybrid DC-DC converter combines the boost and flyback topologies to produce a semi-sinusoidal output current and to achieve the high step-up objective. A full-bridge DC-AC converter controlled with low-frequency switching techniques is then used to convert the current into sinusoidal form and to feed power to the grid with unity power factor. The overall efficiency of the designed system is high due to the losses of both stages are reduced. In this paper, the circuit operating theory of the proposed PV inverter is firstly addressed then an $80 \mathrm{~W}$ prototype system is designed and built. The feasibility and effectiveness of the proposed circuit are confirmed with some simulation and experimental results.
\end{abstract}

Keywords: photovoltaic module, flyback converter, full-bridge DCAC converter, grid connected PV inverter system

Classification: Electron devices, circuits, and systems

\section{References}

[1] Energy and Power, "IEEE Aerospace and Electronic Systems Magazine," Jubilee Issue, pp. 19-26, 2000.

[2] A. Nasiri, S. A. Zabalawi, and G. Mandic, "Indoor Power Harvesting Using Photovoltaic Cells for Low-Power Applications," IEEE Trans. Ind. Electron., vol. 56, no. 11, pp. 4502-4509, Nov. 2009.

[3] C. Rodriguez and J. D. K. Bishop, "Organic Architecture for Small- to Large-Scale Photovoltaic Power Stations," IEEE Trans. Ind. Electron., vol. 56, no. 11, pp. 4332-4343, Nov. 2009.

[4] F. Giraud and Z. M. Salameh, "Steady-State Performance of a GridConnected Rooftop Hybrid Wind-Photovoltaic Power System with Battery Storage," IEEE Power Eng. Rev., vol. 21, no. 2, p. 54, Feb. 2001.

[5] T. Shimizu, K. Wada, and N. Nakamura, "Flyback-Type Single-Phase Utility Interactive Inverter With Power Pulsation Decoupling on the DC Input for an AC Photovoltaic Module System," IEEE Trans. Power Electron., vol. 21, no. 5, pp. 1264-1272, Sept. 2006. 


\section{Introduction}

With the ever growing concern about global warming, environmental pollution, and the rising cost of fossil fuels like oil and coal, there is a greater interest in developing distributed generations (DG) with renewable energy sources (RES) to meet the growing energy demand. It has been well accepted that DG and RES technologies offer the promise of flexible, clean, abundant energy gathered from self-renewing resources. Of these renewable energy sources, photovoltaic (PV) technologies are becoming cost-effective today in an increasing number of markets, and are making important steps to broader commercialization [1]. In addition to central PV inverter systems which are normally designed with relatively high power ratings, the application of small dispersed PV complementary energy systems is another rapidly growing area $[2,3]$ and is developed toward architectures consisting of a number of PV inverters with relatively small power ratings and which can be incorporated into roofing materials or dispersedly installed at any location in the building near an outlet. The number of PV module in the applications of this kind is around 1-5 per inverter, and there will be as many inverters as the application needs $[4,5]$. However, the key to the success of applying small dispersed PV energy systems is a simple, low cost and high performance inverter. In this paper, a novel PV inverter of this kind is proposed and experimentally tested.

\section{Operating principles of the proposed PV inverter}

\section{A. Circuitry and control arrangements}

As shown in Fig. 1(A), in addition to a conventional boost-type DCDC converter and a full bridge DC-AC converter, the proposed PV inverter system includes an extra secondary winding, $N_{s}$, a diode, $D_{2}$, a filtering capacitor, $C$, to form a boost type flyback configuration and to increase the efficiency and output voltage gain with a coupled inductor. A low pass filter is constituted by a clamping capacitor, $C_{\text {clamp }}$, connecting in series with, $C$, and the output inductor, $L_{s}$. By cascading the output voltage, $V_{C c}$, of the boost converter and the output voltage, $V_{c}$, of the flyback converter, a high output voltage, $V_{d}$, is easily obtained. In addition, there is a low voltage stress imposed on the power switch and diode as well as on the output capacitors compared to that of conventional boost converters. The low side capacitor, $C_{\text {clamp }}$, functions as an output capacitor and a snubber capacitor to suppress the voltage spike on $Q_{1}$ during the turn-off transient period, which also recycles the leakage energy in the coupled inductor. In this design, a power switch, $Q_{1}$, with low voltage rating is used to reduce conduction loss, and as a result the overall efficiency can be significantly improved. For the switching control of the proposed PV inverter system, a semi sinusoidal pulse width modulation (PWM) method is utilized. The duty cycle of the switching device, $Q_{1}$, is obtained from comparing a saw-tooth voltage waveform, $V_{t r i}$, and a half-cycle sinusoidal voltage waveform. By setting the peak value of the current flowing through $Q_{1}$ to be proportional to the grid voltage and 
controlling the converter under discontinue current mode (DCM), a half-cycle sinusoidal current waveform can be produced for the output current of the flyback converter, $I_{d}$, which is also proportional to its primary current, i.e., the peak current of $Q_{1}, I_{Q 1}$. In order to convert this half-cycle sinusoidal current waveform into an alternative current waveform, the switching signal for the full-bridge DC-AC converter is made synchronous to the grid voltage, $V_{s}$. The switching devices, $(T A+, T B-)$, of the inverter are turned on during the positive half cycle and the other pair of switches, $(T A+, T B-)$, are turned on during the followed negative half cycle. The output of the full-bridge DC$\mathrm{AC}$ converter is then connected to the grid $\left(V_{s}\right)$ via a low pass filter formed by $V_{C c}$ and $C_{c}$ and $L_{s}$ to achieve feeding unity power factor power to the grid. It should be noted that the overall efficiency of the proposed inverter can be further increased due to the fact that the discharging path of the clamping capacitor, $C_{\text {clamp }}$, does not include the diode.

\section{B. Operating principles}

Before analyzing the proposed PV inverter the following assumptions are made:

1) The main switching device and the two diodes are ideal.

2) The magnetizing current of the transformer is always positive.

3) The leakage inductances $L_{k 1}$ and $L_{k 2}$ are much less than the magnetizing inductance, $L_{m}$.

4) The voltages and currents in the circuit are all periodic under steady state operation.

5) The duty of the main switch is defined as $D$ and the switching period is defined as $T s$.

To analyze the circuit of the proposed PV inverter shown in Fig. 1(A), the
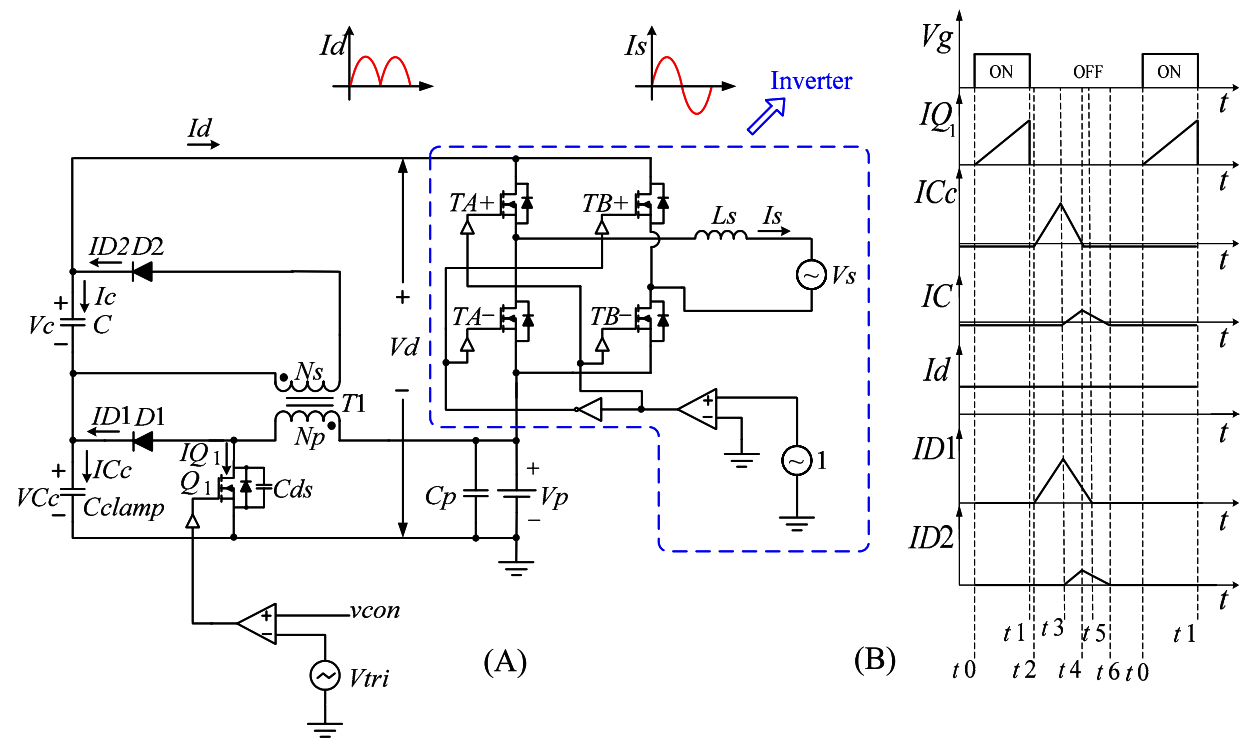

Fig. 1. (A) The circuit of the proposed PV inverter; (B) Typical current waveforms of the converter operated in DCM. 
transformer $T_{1}$ is modeled as a magnetizing inductance, $L_{m}$, and two leakage inductances $L_{k 1}$ and $L_{k 2}$, and an ideal transformer which consists of a turns ratio of $N_{p} / N_{s}$. The typical current waveforms of the converter operated in DCM are illustrated in Fig. 1(B). Fig. 2 (A) to (G) illustrate all operating modes within a positive half-cycle of the grid voltage ( $T A+, T B-$ are on). In Mode (A):t0-t1, $Q_{1}$ on, the clamping capacitor and $\mathrm{C}$ supply energy to the grid; In Mode (B):t1-t2, $Q_{1}$ off, $C_{d s}$ is charging; In Mode (C):t2-t3, the two diodes are turned on. In Mode (D):t3-t4, $I_{D 1}$ is charging the clamping capacitor and $C$ is discharging; In Mode (E):t4-t5, $I_{D 2}$ is charging the flyback capacitor, $C$; In Mode (F):t5-t6, the currents of two diodes are approaching to zero; In Mode $(\mathrm{G})$ :t6-t0, the two diodes are turned off. In the negative half-cycle of the grid voltage the $T A-$ and $T B+$ are turned on.

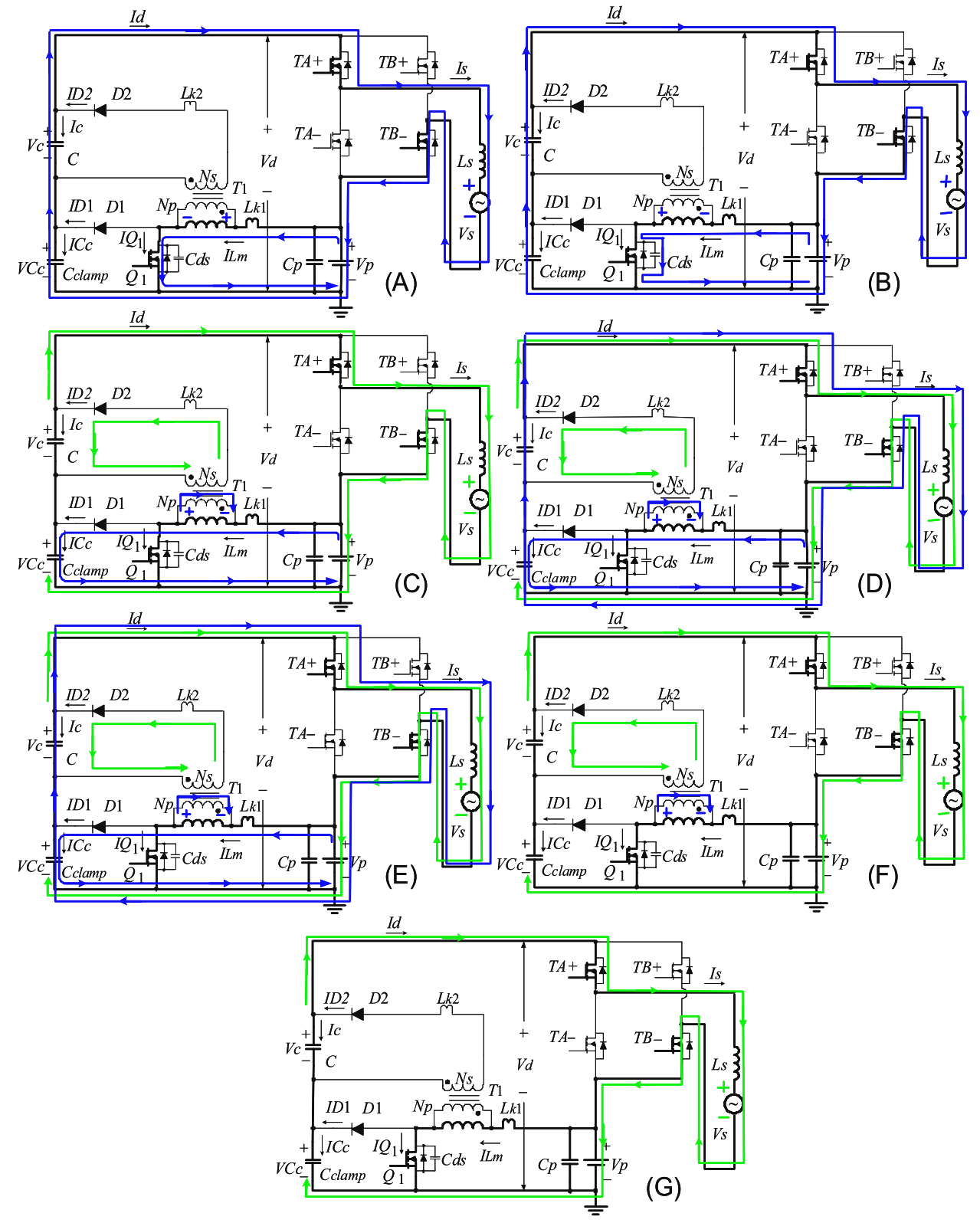

Fig. 2. All possible operating modes (A to $G$ ) within one switching cycle of the converter. 


\section{Specifications of key devices}

Referred to Fig. 1(B) and the equivalent circuit of Mode (C) as shown in Fig. 2, at the time instance t2 when the two diodes, $D_{1}$ and $D_{2}$, are turned on due to forward biased the voltage imposed on the main switch, $V_{D S S}$, equals to the voltage on the output capacitor. As depicted in Fig. 2 (C), when the diode, $D_{1}$, is off and $D_{2}$ is on the voltage imposed on the main switch, $V_{D S S}$, is given by:

$$
V_{D S S}=V_{p}+V_{c} \cdot\left(\frac{N_{p}}{N_{s}}\right)
$$

It follows that $V_{D S S}=V_{C c}$. When the converter is operated in Mode $(\mathrm{G})$, the two diodes, $D_{1}$ and $D_{2}$, are off and the voltage imposed on the main switch, $V_{D S S}$, equals to $V_{p}$, and then the value of $V_{C c}$ can be calculated as follows.

$$
V_{C c}+V_{c}=V_{C c}+\frac{N_{s}}{N_{p}} \cdot\left(V_{C c}-V_{p}\right)=V_{d}
$$

In Eq. (2), the voltage, $V_{d}$, is equivalent to the peak valued of the grid voltage. Rearranging Eq. (2), the voltage on the output capacitor can be mathematically expressed as follows.

$$
V_{C c}=\frac{N_{p} \cdot V_{d}+N_{s} \cdot V_{p}}{N_{p}+N_{s}} .
$$

\section{Experimental system and measured results}

In this paper, an experimental $80 \mathrm{~W}$ PV system is constructed for test purposes. The details of the experimental system are given below.

- Input voltage: $10-40 \mathrm{VDC}$

- Grid voltage/frequency: $110 \mathrm{~V} / 60 \mathrm{~Hz}$

- Switching frequency: $20 \mathrm{kHz} / 5 \mathrm{~V}$ ramp

- PV module: $80 \mathrm{~W}\left(V_{M P P T}=15 \mathrm{~V}\right)$

In hardware implementation of the proposed PV system, there exists two control loops. The outer control loop is a maximum power point tracking (MPPT) controller which is in charge of calculating the operating voltage command for achieving the maximum power operation of the PV module. The inner loop is a voltage regulation loop designed for regulating the voltage of PV module, $\mathrm{V}_{\mathrm{p}}$, to approach the voltage command. To construct the control voltage, $v_{c o n}$, for the converter the output of the voltage controller should be multiplied by a half cycle sinusoidal signal. As can be seen in Fig. 1(A), the trigging signal for the main switching device of the flyback converter is obtained from comparing the control voltage, $v_{c o n}$, with a sawtooth voltage waveform, $V_{t r i}$.

\subsection{Experimental results}

To verify the feasibility of the proposed circuits and design concepts, an $80 \mathrm{~W}$ grid connected PV inverter system is practically constructed. In the hardware system, the PWM switching signals are controlled by the UC3524. 


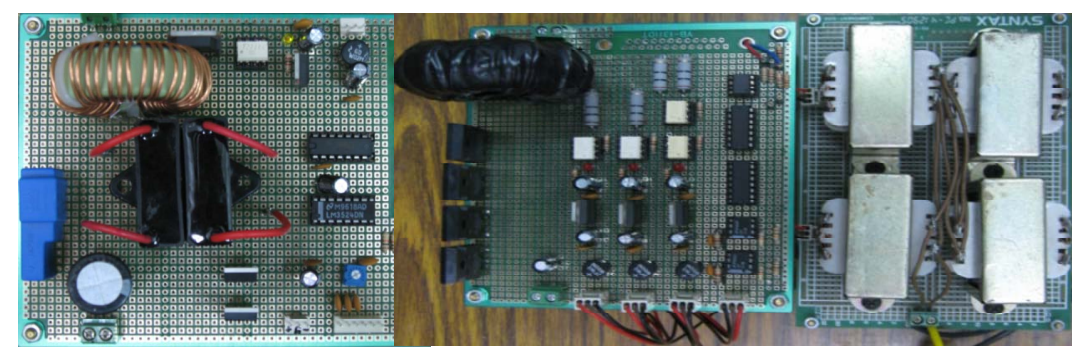

Combined boost and flyback DC/DC converter
Low-frequency switching DC/AC inverter
(A)

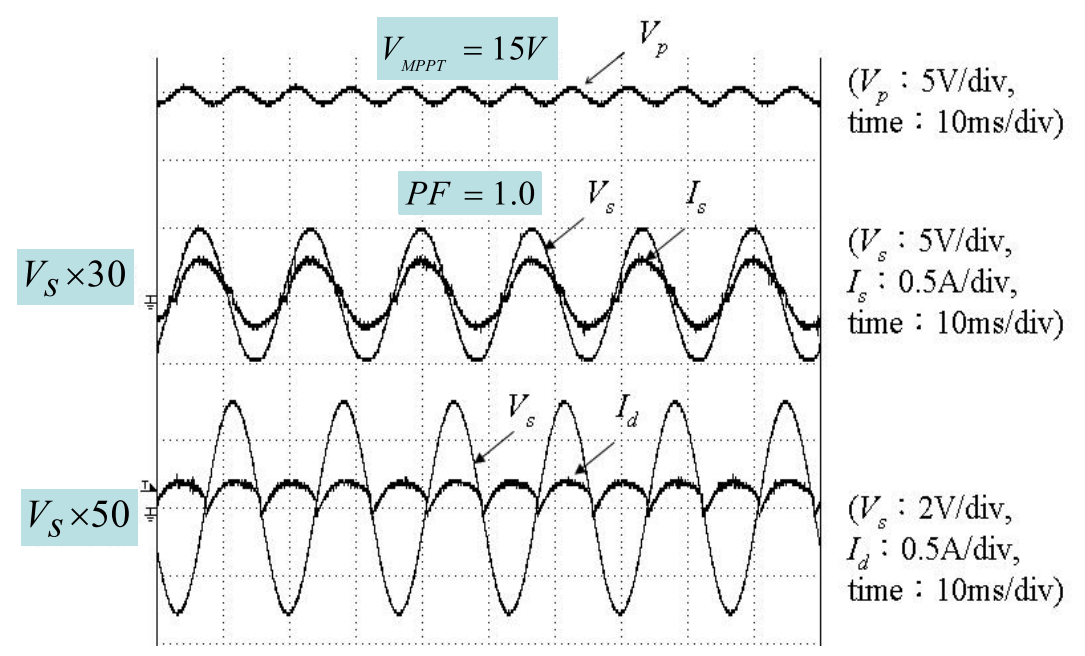

(B)

Fig. 3. (A) The experimentally constructed $80 \mathrm{~W}$ PV inverter system (B) The measured results of the proposed $\mathrm{PV}$ inverter $\left(V_{p}{ }^{*}=15 \mathrm{~V}\right.$, the injected power is controlled at $60 \mathrm{~W})$.

The MPPT controller and the voltage regulator are implemented by using MATLAB real-time control interface. To have a clear picture of the hardware arrangement for the PV inverter system, Fig. 3 (A) shows the photograph of the related circuits. A set of measured experimental results with the output power controlled at $60 \mathrm{~W}$ shown in Fig. 3 (B) verifies the design performance presented above. As can be seen in Fig. $3(\mathrm{~B}), V_{p}$ is regulated at the $V_{M P P T}=$ $15 \mathrm{~V}$ and the unity power factor $(\mathrm{PF}=1.0)$ is achieved.

\section{Conclusion}

In this paper, the concepts of topology design, operating principles, controllers, and experimental results of a novel boost-flyback PV inverter has been presented. In the proposed boost-flyback converter, the leakage energy can be recovered to the output terminal and the voltage stress on the power switch can be significantly reduced. It is important to note that the coupled inductor concept applied to achieve the high step-up voltage gain has been investigated and verified. Based on the measured results, the conversion efficiency is significantly improved (about $95.5 \%$ at 0.75 p.u. output power). The

\section{(c) IEICE 2010}

DOI: $10.1587 /$ elex.7.506 Received February 19, 2010

Accepted February 27, 2010

Published April 10, 2010 
features of the proposed PV inverter can be summarized as high efficiency, high voltage gain, low voltage stress on switching elements, cost effective and simple in hardware implementation. These features are especially important in the low power applications of PV modules. 